Slavica

bruxellensia

\section{Slavica bruxellensia}

Revue polyphonique de littérature, culture et histoire slaves

12 | 2016

Miniatures

\title{
Entretien avec Janusz Szuber
}

\section{Dorota Walczak-Delanois et Anaïs Krawczykowski}

Traducteur : Dorota Walczak-Delanois et Cécile Bocianowski

\section{OpenEdition}

Journals

Édition électronique

URL : http://journals.openedition.org/slavica/1793

DOI : 10.4000/slavica. 1793

ISSN : 2034-6395

Éditeur

Université libre de Bruxelles - ULB

\section{Référence électronique}

Dorota Walczak-Delanois et Anaïs Krawczykowski, « Entretien avec Janusz Szuber », Slavica bruxellensia [En ligne], 12 | 2016, mis en ligne le, consulté le 01 mai 2019. URL : http:// journals.openedition.org/slavica/1793; DOI : 10.4000/slavica.1793

Ce document a été généré automatiquement le 1 mai 2019.

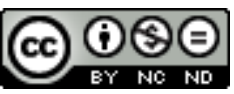

Les contenus de Slavica bruxellensia sont mis à disposition selon les termes de la Licence Creative Commons Attribution - Pas d'Utilisation Commerciale - Pas de Modification 3.0 France. 


\title{
Entretien avec Janusz Szuber
}

\author{
Dorota Walczak-Delanois et Anaïs Krawczykowski \\ Traduction : Dorota Walczak-Delanois et Cécile Bocianowski
}

\section{Présentation de l'auteur}

1 La poésie de Janusz Szuber(né en 1947) est un phénomène d'une beauté particulière. Riche tant en mots, images et sons qu'en récits privés et fragments de la grande Histoire, elle se caractérise par le sentiment d'appartenance à une certaine communauté générationnelle et topographique.

On trouve dans l'œuvre de ce poète originaire de Sanok de belles et réelles amitiés, mais aussi la possibilité de renouer avec soi-même. Tous les recueils de Janusz Szuber sont marqués par la sensibilité à la beauté du monde, à la répétition du destin tout comme au caractère unique de l'individu. C'est un phénomène poétique qui impressionne d'un côté par le ton juste de ses mots et la maestria de son savoir-faire poétique, de l'autre par son audace, son piquant et son registre linguistique particulièrement étendu.

Contemporain des poètes de la "Nouvelle Vague », ses poèmes ont fini dans ses tiroirs pendant vingt-sept ans ; ses débuts tardifs n'ont eu lieu qu'en 1994, lorsqu'il s'est décidé à publier progressivement son œuvre.

Janusz Szuber est l'auteur des recueils suivants : Paradne ubranko i inne wiersze (Habits de parade et autres poèmes, 1995), Apokryfy i epitafia sanockie (Apocryphes et épitaphes de Sanok, 1995), Pan Dymiacego Zwierciadła (Le Maître du miroir qui fume, 1996), Gorzkie prowincje (Provinces amères, 1996), Srebrnopióre ogrody (Les jardins aux plumes d'argent, 1996), Śniąc siebie w obcym domu (Se rêver dans une maison inconnue, 1997), o chłopcu mieszajacym powidła (Sur le garçon qui mélangeait de la confiture de prunes, 1999), Biedronka na śniegu (Une coccinelle sur la neige, 1999), 7 wierszy/ Gedichte (7 poèmes, 1999), Okragte oko pogody (L'œil rond du temps, 2000), Z zóttego metalu (D’un métal jaune, 2000), 19 wierszy (19 poèmes, 2000), Las w lustrach/ Forest in the Mirrors (Forêt dans les miroirs, 2001), Lekcja Tejrezjasza (La leçon de Tirésias, 2003), Glina, ogień, popiół (Terre, feu, cendre, 2003), Tam, gdzie niedźwiedzie piwo warza (Là où les ours brassent de la bière, 2004), Czerteż (* Czerteż est le nom d'un village dans les Carpates, N.d.T., 2006), Pianie kogutów (Le chant des coqs, 2008), Wpis do ksiagg wieczystych (Inscription dans le livre foncier, 2009), Wyzej, nizej, 
juz / Plus haut, plus bas, ça y est (2010), Powiedziéc. Cokolwiek (Dire. Peu importe quoi, 2011), Entelechia/ Entelequia (2012), Antologia (Anthologie, 2013), Tym razem wyránnie (Cette fois clairement, 2014); en prose poétique: Mojość (« Mienneté », 2005, 2012) et le recueil de limerick Emeryk $u$ wód (Emeric aux bains, 2012). Il a écrit entre 2000 et 2001 le cycle de feuilletons Czytane na prowincji ( $\mathrm{Lu}$ en province, dans le journal RZECZPOSPOLITA, supplément PlusMinus).

Ses poèmes ont été traduits en plusieurs langues. Des choix de poèmes ont été publiés en anglais, en serbe, en ukrainien et en allemand.

Janusz Szuber a reçu le Prix Poétique Kazimiera Iłłakowiczówna (1996), Le Prix de la Fondation de la Culture (1999), Le Prix de la Fondation Władysław et Nella Turzański (Toronto), le Prix Poétique K. I. Gałczyński Orfeusz (2015).

Il a été l'initiateur et le cofondateur de nombreux projets promouvant la littérature, il a notamment cofondé l'association Korporacja Literacka et les éditions Cahiers Acta Pankoviana. Il a publié de nombreux articles et essais dans des revues, des ouvrages collectifs ainsi que des préfaces.

\section{Entretien}

\section{Première partie : une histoire personnelle - une histoire parmi les autres}

DOROTA WALCZAK-DELANoIS : Je suis décontenancée. Je me prépare à cet entretien depuis longtemps et pourtant, face à l'immensité du «contenu» et de la «forme» dans votre $œ u v r e$, je me sens désarmée. Vos lecteurs ainsi que tous les curieux doivent faire un choix difficile. Vous rendez-vous compte de la richesse de votre création qui, des tiroirs de votre appartement à Sanok arrive à toucher vos lecteurs au sens propre comme au figuré ?

Janusz Szuber : Dans mon cas, il existe un certain malentendu. La date de ce qu'on appelle mes débuts, 1994, est décisive, bien que mes textes poétiques datent de la fin des années 1960. Beaucoup d'entre eux ont été soumis à une sélection ou ont été détruits lors de leurs réécriture et correction, d'autres se sont trouvés dans le «Pentateuque » du début. Le nombre de mes poèmes publiés s'élève à plus de 500 ; ils recouvrent une période d'écriture active allant de 1968 à 2014. Mes débuts sont donc en quelque sorte un bilan de mon œuvre qui n'avait pas vu le jour depuis plus d'un quart de siècle. Ce n'est qu'après avoir publié mes poèmes pendant plus de dix ans, au moment où mes tiroirs se sont complètement vidés et que leur problème a cessé d'exister, que je me suis senti libre.

ANAïs KRAWCzYKowsKı: Revenez-vous souvent à vos cinq premiers volumes qui constituent le "pentateuque » (Paradne ubranko $i$ inne wiersze [Habits de parade et autres poèmes], Apokryfy i epitafia sanockie [Apocryphes et épitaphes de Sanok], Pan Dymiącego Zwierciadła [Le Maître du miroir qui fume], Gorzkie prowincje [Provinces amères], Srebrnopióre ogrody [Les jardins aux plumes d'argent]) ?

J. S. : J'y reviens s'il le faut: si je suis obligé de choisir ou de me prononcer dans les entretiens ou les interviews que je donne. De moi-même, non. Les textes ne peuvent tout de même pas être corrigés, ils vivent leur propre vie. Si je dois consacrer du temps à la lecture, je préfère lire quelqu'un d'autre que moi-même... La sélection qui a accompagné le processus de rédaction du « Pentateuque » des débuts a été encore plus sévère lors de la rédaction du choix de poèmes $O$ chłopcu mieszającym powidła (Sur le garçon qui mélangeait de la confiture de prunes). Le choix des poèmes constitue un 
élément très important de mon écriture. La première sélection est celle de la composition d'un volume à partir de plusieurs autres, sélectionnés de manière critique. Le choix de poèmes naît ainsi, et c'est justement avec ce choix de poèmes que je m'identifie le plus. Tout ce processus mène à un choix suprême qui est pour moi, auteur, le plus important et le plus représentatif. Jusqu'à présent, ces choix de poèmes s'intitulent Le Chant du coq et Anthologie, publiés aux éditions Hachette.

A. K. : Vos poèmes scintillent par leurs jeux avec les références, les contextes et les allusions (plus ou moins visibles et lisibles) aux grands auteurs comme Czesław Miłosz, Zbigniew Herbert, Aleksander Wat, pour n'en citer que quelques-uns. Dans le poème «Korporacja Umarłych Poetów »(Corporation des poètes morts), vous citez entre autres Bohumil Hrabal. Nous devinons que c'est un monument à la mémoire des poètes qui ont disparu. Quelle est la part du patrimoine que vous leur devez?

J. S. : Dans un certain sens, je leur dois tout. Tout d'abord, l'émerveillement face à leur œuvre, face à la beauté de leurs poèmes. Ensuite, il s'agissait d'observer : comment ontils fait? Chaque poète est un autodidacte, dans le sens où il désigne lui-même son maître, et comme dans l'artisanat traditionnel, il doit tout apprendre de son maître ; mais contrairement au métier d'artisan, le poète ne peut pas copier son maître. La poésie, la littératurese servent d'un matériau très spécifique qui d'un côté est quelque chose d'extraordinaire, et de l'autre, la substance la plus utilisée, exploitée, parfois déformée qui soit. Comment garder la maitrise dans l'utilisation de la matière de la langue ? On cherche des maîtres dont on peut devenir l'apprenti, en gardant en tête la spécificité du matériau, en ayant conscience que le plagiat est le péché le plus grave. Deux livres m'ont guidé durant mon adolescence : Cosmos de Witold Gombrowicz et Le Procès de Franz Kafka, mais cela ne signifie pas qu'il faut penser ou écrire comme Gombrowicz ou Kafka. Se référer aux maîtres ne signifie pas qu'il faut les imiter. Depuis des dizaines d'années, je suis un grand amateur de l'œuvre de Zbigniew Herbert, mais je pourchasse sans pitié tous les " herbertismes » dans mes textes et j'espère que je ne me réfère pas à son écriture comme le ferait un singe, de façon directe. La vigilance permanente est indispensable pour éviter cette faute.

A. K. : Pour rester dans le prolongement de la question précédente, vous avez étudié les lettres polonaises à l'Université de Varsovie, vous êtes membre du Pen Club et de l'Association des Écrivains Polonais (SPP). Dans quelle mesure votre œuvre est-elle marquée de façon solennelle par la culture littéraire de votre patrie ?

J. S. : Si la division simplifiée entre le courant de la constatation et celui de la continuation est légitime, je serais content de me retrouver dans le courant de la continuation, que je comprends comme une prise de distance consciente par rapport aux pratiques saisonnières, souvent commerciales, et à la sensibilité extrême (à la manière des médias), à la déformation idéologique des faits. Pour citer Miłosz: on aimerait avoir dans son jardin littéraire son propre morceau de terrain, ne serait-ce que près de la clôture. J'appartiens à la génération qui lisait beaucoup et goulument. La langue polonaise est ma patrie : de la langue charnelle et juste de l'époque de la vieille Pologne au contemporain littéraire d'aujourd'hui, avec un fort accent sur les écrits des Lumières, et l'omission ostentatoire de la littérature de la Jeune Pologne à cause de son vocabulaire indécis, vacillant et prolifique. 
A. K. : Vous consacrez beaucoup de place à l'histoire racontée dans différentes biographies parsemées dans vos poèmes, dans vos dédicaces, dans vos descriptions de lieux. Est-elle lisible pour les autres? Pensez-vous qu'elle ne constitue pas un problème?

J.S. : Cela dépend comment nous traitons l'histoire, si elle est comprise dans des catégories plus générales, sociologiques et abstraites, si elle se déroule à travers les gens et leurs destins singuliers, entremêlés dans le temps. Un homme peut être le sujet de divagations abstraites, mais il n'aura plutôt pas sa place dans la poésie. Mes héros vivent dans un temps concret, ils ont ou n'ont pas la conscience que le fait d'être a lieu dans des réalités en apparence incompatibles : une pour tout le monde et une à réaliser. On peut bien sûr inventer certaines situations et personnes à l'usage des poèmes, si cela est habilement fait. Ne rejetons pas cela. Cependant, les affaires humaines et universelles peuvent être montrées via des êtres concrets, prises dans un temps et un lieu saisissable. Il s'agit de saisir quelque chose de ce qu'on appelle la vie, qui est, en principe, insaisissable. En écrivant, en évoquant dans le souvenir des personnes précises, je ne crée pas une chronique régionale. Ces biographies peuvent aussi servir à quelque chose qui dépasse le personnel. Mais bien sûr, je les déchiffre à travers moi, en limitant leur autonomie originelle.

A. K. : À l'époque de l'Union Européenne et de la globalisation, plaidez-vous, à travers vos poèmes, pour la reconnaissance de l'identité polonaise?

J. S. : Je ne plaide pour rien du tout. La poésie est un des éléments qui construit l'identité. La question de la langue et de ce qui constitue son fondement inamovible est la base de cette identité. Si les poèmes fonctionnent, et j'ai la chance qu'ils fonctionnent aussi dans d'autres langues, cela veut dire que l'aspect local ne dérange pas pour une réception universelle. Il s'agirait plutôt de l'authenticité de la question de la temporalité, de la superficialité, ou de la profondeur des problèmes évoqués. Les traductions et leur réception, c'est un test important pour la poésie. Les traductions ont cette qualité d'être en fait un nouveau début dans une autre langue. C'est ainsi que l'accent est mis sur l'universalité. On parle alors de certains sujets, primordiaux et irréductibles d'une manière lisible et vérifiable à travers un autre idiome, vérifiable à la fois par l'expérience élémentaire et l'intuition. C'est un argument pour une entente possible au-delà de la communauté nationale.

D. w-D. : Je reviens à Miłosz et à votre histoire. J'ai l'impression que quand Miłosz dit «CELA », vous, poète en dialogue avec Miłosz, vous répondez «RIEN ». Le rien comme catégorie qui apparaît dans vos histoires poétiques. A quel point ce «RIEN »est-il important ? Comment le placer par rapport à la densité des histoires racontées ? Est-ce une recherche d'équilibre, la justification "Ce n'est rien... », ou la naissance de rien, de "l'écume de la mer»?

J. S. : Nous parlons autrement du monde quand on considère le dénudement comme un principe de base, et autrement lorsqu'on perçoit sa valeur au niveau de la sensualité. À vrai dire, c'est une question pour un expert en philosophie. Il vaut mieux qu'il y ait un "quelque chose " plutôt qu'il n'y ait pas ce «quelque chose». Nous savons que la nature de ce qui est se montre très éphémère. Plus elle est éphémère, plus nous nous rendons compte du caractère commun de ce «rien». Il ne s'agit pas ici d'une sorte d'autodéfense à tout prix. Le temps passe, et reste un texte plus ou moins abouti, quelque chose que nous avons fait dans la langue. Apprivoiser le «rien » à travers la richesse sensuelle de ce qui est sans essayer de noyer l'un dans l'autre. D'un côté, nous avons l'opulence et la beauté du monde et les possibilités de la langue qui nomment cette situation, et d'un autre, le pôle opposé, une page avec l'inscription « rien ». Pour 
se rendre compte du "principe de ce rien", il faut faire quelque chose. Je citerai comme illustration mes deux poèmes «Xawery » (Xavier) et « Koda tamtego wiersza » (La Coda de l'autre poème). Et je ne voudrais pas répondre à cette question à l'aide de ce qu'on appelle le nihilisme, car il ne s'agit pas de cela ici. Chaque conversation avec celui qui écrit des poèmes est en fait un substitut. Il écrit, une fois bien, une fois moins bien, créant une sorte d'autobiographie à sa mesure.

D. w-D.: Un de vos volumes contient un poème intitulé "Marian Pankowski », qui a été écrivain et professeur de notre université. Avez-vous lu tout l'œuvre de Pankowski ? ... et?

J. S. : Oui, j'ai lu Pankowski pendant des années. C'est mon maître de langue, une langue enracinée dans le parler local et dans cet idiome spécifique qu'il utilisait non parce qu'il venait de cette région, mais en tant qu'avant-gardiste, ce qui m'a toujours impressionné. On peut être un écrivain moderne et utiliser en même temps des expressions boiteuses des milieux fortement conservateurs, provinciaux; Pankowski me l'a appris, ce qui ne veut pas dire que j'écris « à la Pankowski».

J'étais surtout fasciné par les textes mêmes de son œuvre, et seulement après, par tout ce qui va avec. Nous avons été amis pendant longtemps, ce dont témoigne notre correspondance publiée il y a peu. J'étais ami avec son frère Zygmunt, qui a merveilleusement inspiré, par ses bons mots, de nombreux et intrigants fragments de l'œuvre de Marian. J'ai été l'auteur du choix de poèmes intitulé Ma parole provinciale. Je suis resté en contact avec sa famille de Sanok et jusqu'à la fin, nous avions un contact téléphonique avec Marian Pankowski. Tout cela pourrait être le sujet d'une autre longue conversation.

D. w-D. : Je dois également vous poser une question à propos de Kalman Segal. Comment avez-vous abordé son œuvre?

J. S. : Dans la période de l'entre-deux-guerres, à Sanok, le Gymnase pour garçons du nom de la Reine Zofia était un lieu qui aiguisait et dévoilait les talents à venir. Trois jeunes garçons particulièrement talentueux l'ont fréquenté, à peu près à la même époque : Bohdan Ihor Antonycz, Marian Pankowski et Kalman Segal - un Ruthène (un Lemko), un Polonais et un Juif. C'est fascinant, car Sanok était une petite ville de province. J'ai la satisfaction d'avoir fréquenté les mêmes bancs qu'eux des années plus tard. Dans l'œuvre de Kalman Segal, le Sanok d'avant-guerre existe et il est difficile de regarder cette ville aujourd'hui sans tenir compte du regard de Kalman Segal et de son contact littéraire avec la ville. Antonycz, Pankowski et Segal sont les héros de mes textes qui non seulement les évoquent, mais tentent d'interpréter leur fonctionnement dans ma conscience en tant que représentant des générations suivantes.

D. w-D.: Vous vous êtes lié d'amitié avec beaucoup d'artistes et d'écrivains. Y a-t-il quelqu'un avec qui vous voudriez lier cette belle relation qui est mi-intime, mi-officielle, car solennelle et sacrée?

J. S. : Il me semble qu'il est impossible de planifier une amitié. Il arrive qu'on rencontre quelqu'un et qu'après quelques phrases déjà, se crée un lien spécifique. Une seule conversation suffit parfois entre deux personnes qui écrivent, surtout si elles écrivent de manière semblable. On a alors l'impression que cette connaissance dure depuis toujours. Dans ma vie, j'ai rencontré beaucoup de gens intéressants qui n'ont pas forcément écrit ou peint, et ces rencontres se sont avérées d'une valeur exceptionnelle. J'ai beaucoup appris de mes amis. J'aimerais ici en mentionner un, qui n'est pas un contact littéraire. Il s'agit de feu mon ami Romuald Biskupski, un spécialiste de l'art 
orthodoxe et de la littérature russe, en particulier de celle d'Osip Mandel'štam. Mes contacts avec lui étaient précieux, aussi par rapport à ses travaux érudits.

D. w-D. :Chacun de vos volumes est un récit poétique et beaucoup de ces récits se rencontrent. Combien existe-t-il de versions de votre propre histoire ? Peut-on apprendre à raconter l'inexprimable, ainsi que la difficile expérience de soi-même, des autres et du monde?

J. S. : Ce que vous avez divisé en plusieurs pistes dans votre question existe en moi, auteur et poète, comme une réalité non seulement de l'écriture, mais aussi comme réalité très personnelle. J'évoquerai ici Rilke qui considérait que la poésie est le domaine des expériences et non du moment. Il est vrai que le poème naît sous l'influence de tels ou tels sentiments ou, encore pire, grâce à l'inspiration. Si le Daïmon difficilement exprimable devait avoir une forme, ce serait alors une expérience. Et que nous nous exprimions nous-mêmes à travers les autres? Ce serait maladif si l'auteur s'exploitait sans cesse lui-même dans un sens littéral. Dans mes recueils, ma personne est "empruntée » dans un certain but, tout comme les autres personnes qui me déchargent du problème de l'expression sans gêne. Ces personnes sont-elles d'accord? $\mathrm{Vu}$ la relation qui règne en littérature, elles n'ont pas d'autre choix.

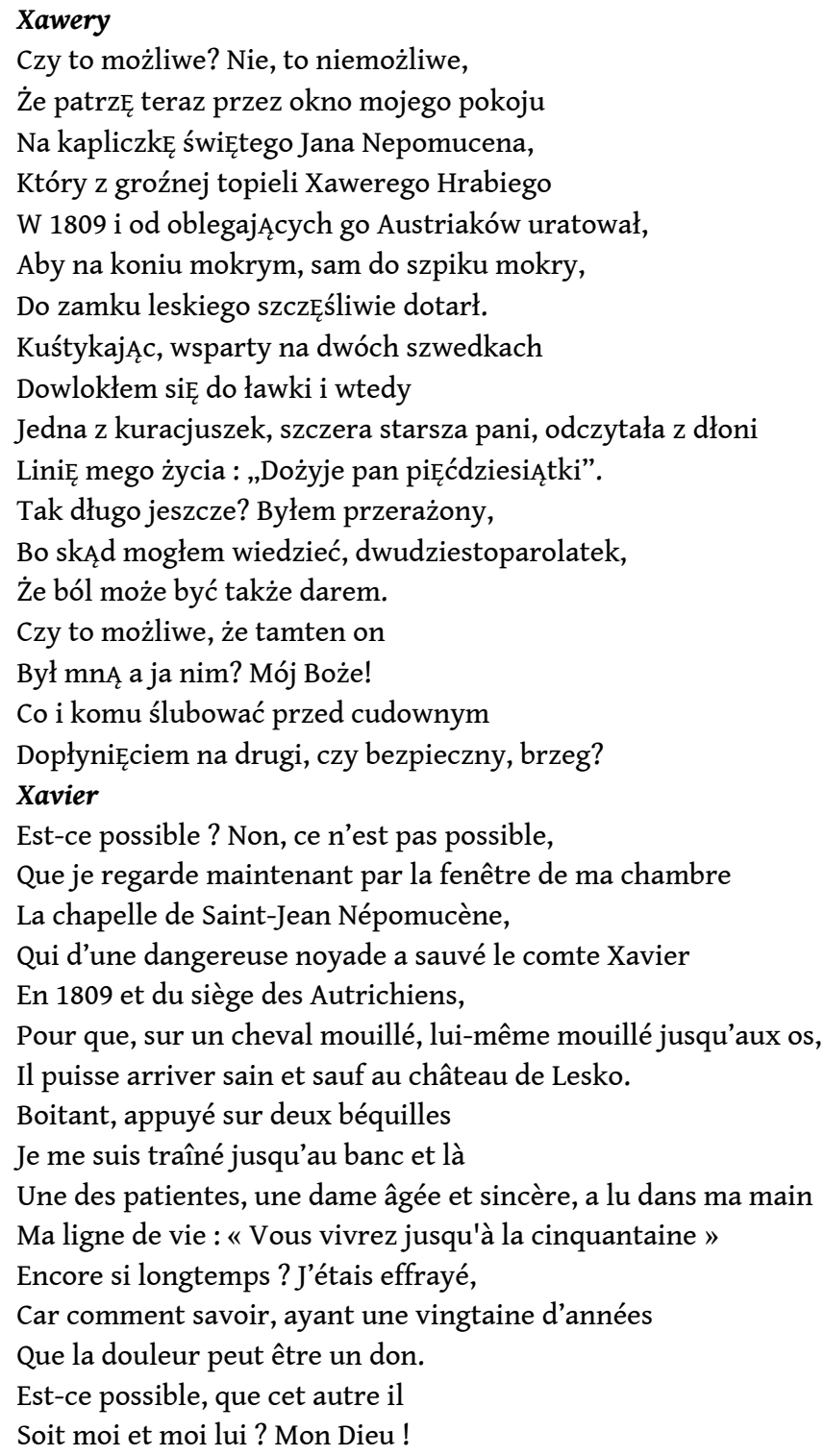


Quoi et à qui prêter serment avant l'arrivée

Merveilleuse sur l'autre rive ou sur la rive sûre?

\section{Deuxième partie : microcosme}

D. w-D. :Dans la multitude des couleurs des mondes que vous présentez, il y a des microcosmes particulièrement beaux et importants. II me semble que, parfois, ils ressemblent à une mise en abyme, à l'image des poupées russes emboîtées, par exemple dans la métaphore si forte (et si belle) des «pivoines blanches des cygnes » dans l'image de «Mgły » (Brouillards) dans ce volume concret et solide qui fait partie de la plus grande des «poupées russes». Construisez-vous parfois votre microcosme à l'instar d'une ville poétique ? Comme un parallèle à la ville de Sanok ?

J. S. : Le matériau de base du recueil est le poème individuel. Quant au volume composé, il a ses propres sujets, mots, métaphores qui s'entrelacent, et s'unissent en un tout. De quelques volumes naît un choix de poèmes qui a aussi ses propres éléments de construction. Les poèmes, dans un autre voisinage, prennent des significations et des nuances différentes. C'est comme la préparation d'une exposition de travaux actuels ou celle d'une rétrospective. Tout dépend sur quels principes on l'agence. Nous pouvons également nous servir d'une métaphore architecturale: il y a des mansardes, des rajouts. La ville est une métaphore très vénérable qui entre dans les régions de la métaphysique, l'équivalent de la structure intellectuelle. Cette dernière se rapporte au processus de « civiliser la nature ». La ville suit ses propres lois de l'espace. Traduire ces mécanismes en poésie serait alors un exercice de géométrie ou de trigonométrie... La poupée russe rend de manière très suggestive la création d'un poème, d'un volume ou d'un choix de poème, mais en réalité aucune nouvelle structure ne se réfère à la structure précédente de manière identique comme dans le cas des poupées russes. Il s'agit plutôt du déménagement d'un tableau dans une salle d'exposition. Tous les éléments sont minutieusement choisis pour que les significations cachées nous parlent davantage. Le poème se construit de façon à ce qu'il soit, dans la couche du récit, relativement simple et qu'il se laisse raconter. Les ressources fondamentales d'un poème sont celles de l'expérience et non de l'émotion, et ce qui résulte de l'expérience est d'habitude transparent. Dans la couche plus profonde se cache ce qui est compliqué, et il ne s'agit pas de l'illustration d'une thèse, ce serait trop simple. Ici, on peut instinctivement atteindre des choses cachées plus profondément, «sous la doublure ». Cependant, le poème ne peut pas n'être que l'illustration d'une thèse philosophique, puisque toutes les constatations devraient avoir leur source dans l'expérience qui est ensuite reprise dans la langue - la matière primaire exerçant aussi des fonctions, également utilitaires. Le son, la couleur, la forme existent pour eux-mêmes. Il en va autrement de la langue, alourdie par ses différentes fonctions. Il ne s'agit pas d'épurer la langue de la poésie qui existerait alors seulement dans la littérature et pour la littérature. C'est impossible et inutile. Il faut être vigilant...

A. K. : Vous aimez aussi les formes moins «contenantes»: apocryphe, épitaphe, haïku, limerick. Est-ce que cela représente un grand défi pour un poète qui a la veine d'un conteur, de tenir dans ces limites?

J. S. : Quiconque traite sérieusement l'écriture de poèmes doit passer par l'école des maîtres, ce qui impose une rigueur formelle. Se servir de pastiches, par exemple, ne signifie pas se limiter; au contraire, cela stimule l'imagination. J'en parle dans mon poème "Ars Poetica»: l'imagination devrait se tenir à la réalité vérifiable. Vous constatez que j'ai une veine de conteur. Le conteur doit s'accrocher à quelque chose, 
alors je m'accroche à une sorte de langage. L'acte de nommer, en parlant simplement, qui se déroule dans un lieu et un temps définis devrait, selon moi, être vérifiable. J'évoquerai ici le nom de Zbigniew Herbert, mon maître, qui s'est moqué d'un piano à queue dont on jouait sur le Mont Blanc... Il $\mathrm{y}$ a eu des périodes en littérature où l'imagination rompait avec la réalité, ce que je suis capable d'apprécier et de respecter, mais mon tempérament est comme il est, ma poétique est telle que j'ai réussi à l'élaborer, bien enracinée dans la réalité palpable. La civilisation contemporaine, et surtout les domaines qui se servent de nouveaux moyens de communication, créent des possibilités illimitées : on est partout et en même temps nulle part, tout est possible. Je ne critique pas cela, mais je voudrais que dans mes poèmes, le monde ne se dilue pas dans l'horreur d'une science-fiction. Le problème de notre temps repose non seulement sur le fait que tout est permis, mais aussi que tout peut être réalisé d'une certaine manière par une multitude de simulations. La réalité virtuelle peut expulser ce qui nous définit en tant qu'êtres matériels, biologiques, à vrai dire animaux. Cela nous mène directement dans les cercles de l'enfer, et la crainte des romantiques, exprimée par Juliusz Slowacki, commence à se réaliser : «Et il me semble que le vent me disperse ». On devient moins concret; nous sommes des « êtres cheminant vers la mort » et nous ne changerons rien à cela. Dans le temps, les objets passaient d'une génération à l'autre, ils étaient porteurs de mémoire et déterminaient une continuité. Aujourd'hui, les objets ont la vie courte. Ils sont interchangeables. La mode devient le patron de la civilisation. La mode saisonnière entre aussi dans les domaines artistiques et intellectuels, et cela mène à la perte de caractéristiques. Nous ne nous opposons pas à la civilisation. Je ressens d'une manière poignante le rien omniprésent. Pour ne pas adapter de pose nihiliste, je m'accroche, comme à une bouée de sauvetage, à quelque chose de palpable, qui donne sa base au mythe. Le mythe est le partenaire du rien. Surtout ne pas se laisser posséder par ce courant gourmand et conjoncturel.

D. w.-D. :L'érotisme constitue chez vous un microcosme à part. La masculinité mise face à la féminité, les rencontres du corporel dans les tout petits détails, dans des portraits non évidents. Vos poèmes sont très sensuels. Et beaux. Le microcosme du corps devient-il macrocosme? Peut-il durer?

J. S. : L'érotisme, ainsi que tout ce qui en ressort, doit renforcer ce que j'ai déjà évoqué. Nous pouvons bien sûr l'amener au niveau des procédés biochimiques ou biophysiques. L'érotisme, dans certains degrés de compréhension, reste encore lié au bel esprit. Offrir à quelqu'un son cœur touche aujourd'hui à la fois au domaine des émotions, de l'érotisme et de la médecine. La lune observée aujourd'hui par l'homme qui la foule et observe de près sa surface de "pierre ponce » devient courbe, à double sens, elle cesse d'être uniquement lyrique. Peut-être que la culture engloutira ce concept et lui attribuera une nouvelle qualité. Puisque la poésie est un domaine de l'expérience, tout ce qui change au tempo de la physique est soumis à l'évaluation. Trouver l'équivalent laïc de la durée, c'est là qu'apparaît l'érotisme dans mes poèmes (notamment « Próbuję być » [J'essaye d'être] ou "Klara»). Puisque nous mangeons, nous déféquons, nous naissons, nous mourons, nous sentons encore, nous ne pouvons pas être totalement abstraits. Cela se superpose dans certaines existences au sens topographique et culturel. 
D. w.-D. : Vous êtes un mentor, un sage (ne protestez pas, s'il vous plaît), un poète éclairé. Est-ce qu'il vous arrive de conseiller de jeunes gens?

J. S. : Ces formulations qui décrivent ma personne ne la reflètent justement pas. Je ne me sens jamais comme cela, je ne me pense pas ainsi. Peut-être que je fais parfois des oraisons, comme ici dans notre conversation, ex cathedra, mais ce n'est qu'une convention, puisqu'il faut s'exprimer « d'une certaine manière »... J'ai une attitude très critique par rapport à moi-même. Je ne conseille pas les jeunes gens, surtout parce que j'ai devant moi mon numéro de registre national et ma date de naissance. Les temps dans lesquels nous vivons ne sont pas des temps purs.

\section{Troisième partie : trampoline de mots - trampoline d'images}

D. w.-D. : Il y a de la musique dans vos poèmes. Tous vos critiques sont d'accord sur ce point: vous entendez le monde et savez aussi le transposer en musique. II y a des ensembles que vous aimez particulièrement dans les mots qui chuintent, qui grincent. Et vous aimez le jazz. Est-ce que vous entendez vos poèmes? Est-ce que le poème doit être entendu, est-ce que vous vérifiez vos poèmes «à haute voix »?

J. S. : D'ordinaire, le poème en train d'être écrit, qu'il s'agisse d'un fragment du début ou d'une autre version d'une entièreté prévisible, est créé «à haute voix». La musicalité qui l'accompagne module le texte en lui attribuant le caractère de ce poèmeci, et non d'un autre. Dès le début, il est créé comme "prononçable d'une certaine façon ». D’habitude, mes poèmes sont courts, ils dépassent rarement vingt, vingt-cinq vers, donc ils sont relativement faciles à saisir à la fois par la vue mais aussi par l'ouïe. Dans l'intention de l'auteur, ils devraient être une entièreté cohérente, dans son aspect graphique et auditif. C'est pourquoi j'enregistre mes poèmes sur un dictaphone, je les écoute plusieurs fois en les corrigeant jusqu'à l'obtention de l'effet désiré. J'ajouterai au passage qu'une musique a été composée pour quelques dizaines de poèmes qui ont été interprétés à différentes occasions.

D. w.-D. :Dans quelle direction se dirige la flèche du vecteur: de l'image aux mots ou des mots à l'image? Et est-ce que le début, l'étincelle avant l'explosion du poème, ont une signification? Comment le poème naît-il en vous?

J. S. : La seule réponse honnête à cette question pourrait être: je ne sais pas. C'est différent à chaque fois. J'ai écrit plus de 500 poèmes et je suis toujours aussi incapable de dire ce qui se passe en moi au moment de leur création, si ce n'est de raconter une anecdote créée à la va-vite et aux airs de confession sur la façon dont le poème renforce la sensibilité. Il n'y a pas de "visions, étincelles et explosions » dans mon cas, je travaille laborieusement mon poème : saisir quelque chose, s'y accrocher, et lui donner une expression indépendante.

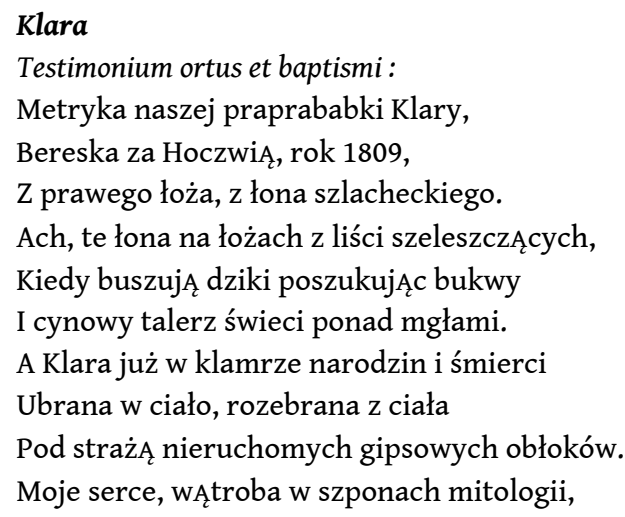


Moje grube jelito, jĄdra testis testimonium.

Moje, twoje, niczyje. Bezradny,

Jak ty, Klaro, coraz bardziej nagi,

Rozpuszczony w powszechnym, prawie przeźroczysty

Wzywam na pomoc zaimek dzierżawczy,

$\mathrm{Z}$ prawideł gramatyki czerpiĄc

Konieczny argument.

Claire

Testimonium ortus et baptismi :

L'acte de naissance de notre arrière-grand-mère Claire,

Bereska après Hoczwia, année 1809,

De filiation légitime, de giron noble.

Oh, ces girons sur des lits de feuilles bruissantes,

Quand les sangliers furètent en cherchant des faînes

Et l'assiette en étain brille au-dessus des brouillards.

Et Claire déjà entre les parenthèses de la naissance et de la mort

Vêtue d'un corps, dévêtue du corps

Sous la garde immobile de nuages de plâtre.

Mon cœur, mon foie dans les griffes de la mythologie,

Mon gros intestin, mes testicules testis testimonium.

Les miens, les tiens, ceux de personne. Désarmé,

Comme toi, Claire, de plus en plus nu,

Dissout dans le tout, presque transparent

J'appelle à l'aide le pronom possessif

Puisant dans les lois de la grammaire

L'argument nécessaire.

D. w-D. : Il y a beaucoup de couleurs dans vos poèmes : si l'on observe la façon dont vous utilisez les couleurs, jouez avec le cercle chromatique, on s'aperçoit rapidement que vous êtes un coloriste exceptionnel. Je sais que vous vous intéressez aussi aux arts graphiques. D'où vient cet intérêt ? Et êtes-vous insensible à un type d'art?

J. S. : Il me semble que j'ai toujours été attaché aux arts plastiques. Cela a commencé tôt pendant mes vacances annuelles chez mon grand-père à Krynica : j'ai passé beaucoup de temps, tous les ans, planté près du peintre Nikifor (Epifaniusz Drowniak) pendant qu'il peignait, et ce primitiviste me fascine jusqu'à aujourd'hui. J'ai eu la chance de voir de mes propres yeux le monde artistique si particulier de Marian Kruczek lorsqu'il venait, de l'Académie des Beaux-Arts de Cracovie, chez mes parents à Płowiec, pour y travailler sur une de ses compositions spatiales. Ces deux artistes, Nikifor et Kruczek, sont à l'origine de ma fascination pour l'art. En grandissant, j'ai passé beaucoup de temps dans des galeries d'art et sur des albums. Moi-même, j'ai beaucoup dessiné et peint jusqu'à mes 18 ans à peu près. Ce n'est que plus tard que je me suis occupé de poésie. J'ai de nombreux peintres parmi mes amis et leurs travaux sont suspendus chez moi, à côté des tableaux des années 1920 et 1930. La collection des œuvres de Leszek Rózga représente une aventure en soi : j'ai rassemblé plus de 90 de ses œuvres, et chaque fois que j'en achetais une, j'avais droit à une conversation et un cours du Professeur.

Je suis de nature plutôt tolérant, mais je réagis de façon allergique à tous types de fantasy, à l'exception des graffiti sur les garages qui utilisent ces motifs.

A. K. : Dans vos poèmes, vous faites allusion à la vie quotidienne, aux rituels du bain, à Internet. Le poète doit-il aujourd'hui prendre en compte la société « accélérée » ? Comment envisagez-vous la société d'aujourd'hui?

J. S. : Il est évident qu'en vivant dans un lieu et un temps donnés, on s'imbibe de son atmosphère et de sa langue. Je m'imbibe de tout ce dont vivent mes contemporains au 
quotidien, ceux avec qui j'ai des contacts, puisqu'il n'y a pas et il ne peut y avoir d'autre «ici » et "maintenant», ce qui ne m'empêche pas de faire des expéditions dans d'autres régions déjà vérifiées et stables, mais sans m'enfermer dans un mausolée et scander des chants historiques dans un Nulle part fantomatique. Je ne peux rien au fait que, tout comme mes contemporains, je suis bombardé des produits de l'information omniprésente et désordonnée. Il est sûrement de plus en plus difficile avec chaque génération d'être indépendant, car les rebellions saisonnières sont liées au marché et assurent des profits conformistes à ceux qui les administrent. Il n'est pas possible de faire semblant que l'on est isolé de son entourage.

A. к. : Un de vos poèmes s'intitule "Andy Warhol », figure emblématique du pop art, un autre, «Bogom tej prowincji »(Aux Dieux de cette province), cite Maradona et Bruce Lee. Peut-on dire que vous tendez à la post-poésie? Est-ce un antidote à l'iconicité de ces personnages?

J. S. : Andy Warhol, c'est en quelque sorte un compatriote. Il n'y a qu'un pas de chez moi à son village lemkovien. Pourquoi lui, Maradona ou Bruce Lee ne devraient-ils être présents que dans la post-poésie ? Et pourquoi devrions-nous feindre d'être différent de la façon dont on nous voit, du point de vue des statistiques et de la physiologie, conscients que bon gré mal gré, nous faisons également partie en quelque sorte de la manipulation générale. La poésie élevée, le sacre du poète face au rythme effréné de la civilisation a l'air bien blême, et pouvoir parler de statut privilégié de la poésie et du poète requiert quantité d'efforts et, disons-le, de se mentir à soi-même. Mais malgré tout cela, cela vaut la peine d'écrire de la poésie, à condition que ce soit de la bonne poésie, c'est-à-dire honnête envers la réalité et la langue dans laquelle elle est écrite.

A. K. : On pourrait dire, en schématisant, que la poésie est la dernière forme d'art élitiste. Vous soulignez l'idée que vos poèmes traitant de sujets liés à la société occidentale contemporaine participent à la diffusion, la propagation de cette poésie dans des milieux non élitistes. La poésie peut-elle être l'affaire de tous?

J. S. : Je répondrai par une chose simple : la publication de la poésie et sa distribution. Quoi que l'on pense de soi et de la poésie, cela ne changera pas des faits primordiaux : la publication et la distribution de recueils de poésie se réalisent à doses homéopathiques. Le système éducatif à l'école n'aide pas à retenir la poésie, à moins qu'elle n'appartienne au genre spécifique des poètes cultes. Les sociologues s'occupent de la description de la civilisation contemporaine de masse et de la globalisation. Dans ce monde commun, il ne reste qu'un petit coin pour la poésie, à moins qu'un nom soit auréolé d'événements médiatiques bruyants comme des scandales ou de grands prix. Écrire de la poésie et pratiquer l'art élitiste repose sur le fait qu'il existe une hiérarchie, quelque chose de plus important, et au pôle opposé, quelque chose d'inférieur, ce qui est fondamentalement un concept offensant pour la société de masse.

\section{Quatrieme partie : hauts vols}

A. к. : On vous pose probablement souvent la question, mais cela vaut la peine de la reposer : qu'est-ce qui vous a retenu de publier plus tôt ? Pourquoi attendre vingt-sept ans ?

J. S. : J'ai commencé à écrire en tant qu'adulte, pendant mes études, après avoir peint pendant longtemps et après une période consacrée à une prose bancale et justement abandonnée. J'ai commencé à écrire de la poésie pour essayer, comme ça, pour voir ce que je valais. Aucune vocation pompeuse. J'ai donné ces premiers essais à lire, sans penser à une éventuelle continuation, à un professeur de poétique et bon critique 
renommé. Malgré son opinion positive, je ne me suis pas décidé à publier. Si je devais publier mes vers, pensais-je, ils devraient remplir ne serait-ce qu'une partie de ma vision de la poésie et avoir une forme assez indépendante et reconnaissable. Je lisais beaucoup de poésie et sur la poésie pour ne pas me laisser séduire par des choses dont je n'étais pas sûr ou qui ne soient que passagères. En théorie, dans le futur je voulais m'occuper principalement de littérature. Dans un futur qui a bientôt radicalement changé : d'un homme de catégorie A à l'armée, en sautant sur les montagnes, je suis devenu son opposé et j'ai atterri dans un fauteuil roulant, ce qui était déjà un succès en soi. Dans cette situation, je devais m'opposer à la réduction de l'infantilisation qu'apportaient avec elles les souffrances. J'ai passé les années suivantes à écrire, en repoussant la publication à plus tard. À l'âge de 47 ans, quand je suis arrivé au constat que je ne savais pas écrire autrement, ont eu lieu mes débuts en retard d'un quart de siècle. Marian Pankowski, qui avait déjà eu affaire à mes poèmes, a écrit la postface du dernier tome du «Pentatheuque ». Au début, je ne révélais pas d'informations sur moi, ma maladie et mon invalidité, de peur qu'on m'accorde un tarif spécial pour cela. J'attendais une confirmation objective. J'ai bientôt reçu une première lettre de Zbigniew Herbert, et Miłosz et Szymborska m'ont également chaleureusement accepté. Après la parution en anglais chez Knopf à New York, Seamus Heaney m'a contacté. Voilà, c'était ça.

D. w.-D. : Je reviens à Marian Pankowski, par sentiment pour une de ses nouvelles intitulée Lekcja latania (Leçon de vol). Je suis impressionnée que vous sachiez voler! Au milieu des nuées qui peuplent si souvent votre œuvre... Voler permet d'avoir un meilleur point de vue sur les choses. Comment apprend-on à voler? Et où aimeriez-vous vous envoler?

J. S. : Je ne nourris pas une haute opinion de mon imagination ni de mes talents. Mon imagination est assez pauvre, elle a besoin de s'accrocher à quelque chose. Ce que vous avez gentiment dit sur le fait de voler, en évoquant Marian Pankowski qui m'est cher, a une origine très prosaïque. Mon père était passionné de tout son être par l'aviation. Encore en secondaire, il avait pris des cours de planeur, il était élève officier à l'école d'aviation à DĘblin ; après la Seconde Guerre mondiale, il a travaillé dans l'aviation et a pratiqué sa passion pendant de longues années. En tant que petit garçon, je m'intéressais au modélisme, aux cerfs-volants à caisson, aux modèles radiocommandés avec moteurs. Ça se comprend, mon père m'emmenait à l'aéroport, je volais dans des planeurs et des avions de sport, ainsi que dans le légendaire biplan Polikarpov Po-2. Voilà ce qui a initié mes vols poétiques.

A. к. : Vos poèmes sont remplis d'éléments concrets, et pourtant, le rêve y occupe une place importante, comme s'il devait équilibrer la matérialité du monde qui nous entoure. Quel est le sens du rêve poétique pour vous : le poète doit-il rappeler au monde qu'il faut aussi rêver? Que, comme chez Calderón, la frontière entre rêve et réalité n'est pas évidente?

J. S. : Les rêves, au même titre que la physiologie biochimique vérifiable en laboratoire, participent à la construction de notre « ici » existentiel. Que Calderón demeure, malgré mes nombreuses lectures anthropologiques (d'anthropologie structurale, puisqu'elle m'a formé en grande partie). Tel qu'on me dit et m'imagine, ainsi je dis et j'imagine. À tous les niveaux de la réalité qui me sont accessibles, par l'utilisation d'une langue ou des langues qui lui sont adaptées. La langue du rêve est particulièrement utile dans les activités qui tendent à saisir la partie perpétuellement éphémère de mon existence. 
A. $\mathbf{k}$ : : Quel sujet n'oseriez-vous pas aborder dans votre œuvre?

J. S. : Comme je le disais, je n'oserais écrire sur ce qui n'est pas vérifiable pour moi, ancré dans une idéologie. J'ai peur, je me répugne et j'essaye d'éviter l'impudence à laquelle mènent les auto-présentations comme toutes les stratégies qui puisent dans la sphère intime de notre vie privée et les révélations qui y sont cachées. Auprès du manque notoire d'autres attraits et capacités, cette impudence, comme je l'appelle ici, est malheureusement immédiatement utile et primée - légitimant, à la Big Brother et les reality shows, l'esthétique de badauds.

D. w.-D. : Merci pour vos poèmes qui - avec votre accord - ont rythmé cet entretien. Une dernière question : Qu'est-ce que le Poète a peur d'oublier?

J. S. : Je ne voudrais pas oublier le temps.

Je pense à moi et je parle de moi comme mon acte de naissance Janusz Szuber me décrit avec l'éventuelle annotation "poète", autrement dit celui qui a été accepté par les anciens de la guilde et a ainsi reçu des droits. Je ne voudrais pas oublier le temps quand il faut dire la fin et poursuivre encore dans l'auto-épigonie, en comptant sur l'indulgence de mon entourage.

2 Nous vous remercions chaleureusement, au nom de la Rédaction et des lecteurs.

\section{INDEX}

Index géographique : Pologne

Mots-clés : Littérature polonaise, poésie polonaise

Index chronologique : XXe siècle, XXIe siècle

\section{AUTEURS}

\section{DOROTA WALCZAK-DELANOIS}

Responsable de la Chaire de Polonais, section de Langues et Littératures modernes, option Slaves, de l’Université Libre de Bruxelles (Belgique) ; Rédactrice en chef de Slavica Bruxellensia

\section{ANAÏS KRAWCZYKOWSKI}

Doctorante en Etudes slaves, Université Libre de Bruxelles 